\title{
BMJ Open The effect of nut consumption on markers of inflammation and endothelial function: a systematic review and meta-analysis of randomised controlled trials
}

\author{
Elizabeth P Neale, ${ }^{1,2}$ Linda C Tapsell, ${ }^{1,2}$ Vivienne Guan, ${ }^{1}$ Marijka J Batterham ${ }^{3}$
}

To cite: Neale EP, Tapsell LC, Guan V, et al. The effect of nut consumption on markers of inflammation and endothelial function: a systematic review and meta-analysis of randomised controlled trials. BMJ Open 2017;7:e016863. doi:10.1136/ bmjopen-2017-016863

- Prepublication history and additional material for this paper are available online. To view these files, please visit the journal online (http://dx.doi. org/10.1136/bmjopen-2017016863).

Received 15 March 2017 Revised 18 0ctober 2017 Accepted 19 0ctober 2017

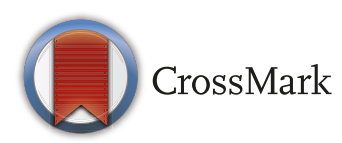

${ }^{1}$ School of Medicine, Faculty of Science, Medicine and Health, University of Wollongong, Wollongong, Australia ${ }^{2}$ Illawarra Health and Medical Research Institute, University of Wollongong, Wollongong, Australia

${ }^{3}$ Statistical Consulting Service, School of Mathematics and Applied Statistics, Faculty of Engineering and Information Sciences, University of Wollongong, Wollongong, Australia

Correspondence to Dr Elizabeth P Neale; elizan@uow.edu.au

\section{ABSTRACT}

Objectives To examine the effect of nut consumption on inflammatory biomarkers and endothelial function.

Design A systematic review and meta-analysis. Data sources MEDLINE, PubMed, Cumulative Index to Nursing and Allied Health Literature and Cochrane Central Register of Controlled Trials (all years to 13 January 2017). Eligibility criteria Randomised controlled trials (with a duration of 3 weeks or more) or prospective cohort designs conducted in adults; studies assessing the effect of consumption of tree nuts or peanuts on C-reactive protein (CRP), adiponectin, tumour necrosis factor alpha, interleukin-6, intercellular adhesion molecule 1, vascular cell adhesion protein 1 and flow-mediated dilation (FMD).

Data extraction and analysis Relevant data were extracted for summary tables and analyses by two independent researchers. Random effects meta-analyses were conducted to explore weighted mean differences (WMD) in change or final mean values for each outcome.

Results A total of 32 studies (all randomised controlled trials) were included in the review. The effect of nut consumption on FMD was explored in nine strata from eight studies (involving 652 participants), with consumption of nuts resulting in significant improvements in FMD (WMD: $0.79 \%(95 \% \mathrm{Cl} 0.35$ to 1.23)). Nut consumption resulted in small, non-significant differences in CRP (WMD: $-0.01 \mathrm{mg} / \mathrm{L}$ $(95 \% \mathrm{Cl}-0.06$ to 0.03$)$ ) (26 strata from 25 studies), although sensitivity analyses suggest results for CRP may have been influenced by two individual studies. Small, non-significant differences were also found for other biomarkers of inflammation.

Conclusions This systematic review and metaanalysis of the effects of nut consumption on inflammation and endothelial function found evidence for favourable effects on FMD, a measure of endothelial function.

Non-significant changes in other biomarkers indicate a lack of consistent evidence for effects of nut consumption on inflammation. The findings of this analysis suggest a need for more research in this area, with a particular focus on randomised controlled trials. PROSPERO registration number CRD42016045424.
Strengths and limitations of this study

- This is the first known systematic review and meta-analysis which examined the effect of nut consumption on inflammation and endothelial function, in studies which isolated the effect of nut consumption.

- The protocol for the review was preregistered, and the review followed the requirements of the Preferred Reporting Items for Systematic Reviews and Meta-Analyses statement.

- Risk of bias was assessed using the Cochrane Risk of Bias Tool, and the quality of the body of evidence was then determined using grading of recommendations assessment, development and evaluation (GRADE).

- The available evidence base for some of the biomarkers explored was small.

- There were variations in the included studies, such as participant health status, nut type and dose and study duration, although these factors were explored in subgroup analyses.

\section{INTRODUCTION}

Chronic conditions such as type 2 diabetes and metabolic syndrome are known to be underpinned by a state of low-grade inflammation, which play a central role in disease progression and in the development of atherosclerosis. ${ }^{12}$ Changes in this inflammatory state can be identified via biomarkers of inflammation including C-reactive protein (CRP) ${ }^{3}$ tumour necrosis factor-alpha $(\mathrm{TNF}-\alpha),{ }^{4}$ interleukin-6 (IL-6) ${ }^{5}$ and the adhesion molecules intercellular adhesion molecule 1 (ICAM-1), vascular cell adhesion protein 1 (VCAM-1) ${ }^{6}$ as well as anti-inflammatory biomarkers such as the adipocyte adiponectin. ${ }^{7}$ Endothelial dysfunction is a central component in the development and progression of atherosclerosis, with brachial flow-mediated dilation (FMD), a non-invasive measure of endothelial 
function, found to be significantly associated with risk of cardiovascular events. ${ }^{8}$

Given that markers of inflammation and endothelial function can indicate changes in disease development and progression, they can be used to explore the impact of consumption of specific foods on health. Nuts contain a wide range of nutrients and bioactive components which may moderate inflammation and the development of endothelial dysfunction, such as alpha-linolenic acid, L-arginine, fibre and polyphenols. ${ }^{9}$ Habitual nut intake has been associated with reduced risk of cardiovascular disease ${ }^{10}$ decreased incidence of the metabolic syndrome ${ }^{11}$ and decreased risk of diabetes. ${ }^{12}$ Clinical trials have previously explored the effects of nut consumption on markers of inflammation and endothelial function, with a range of effects observed. ${ }^{13-22}$ A systematic review and meta-analysis would consolidate and appraise the quality of this body of evidence, providing greater clarity where inconsistencies are observed. Even so, the effort is ongoing. For example, a recently published systematic review did not report significant effects of nut consumption on $\mathrm{CRP}^{23}$ but did not include results of the large PREvención con DIeta MEDiterránea (PREDIMED) study. ${ }^{24}$ It is also possible to consider FMD as an outcome which this previous review did not consider. The aim of the review reported here was to examine the effect of nut consumption on markers of inflammation and endothelial function (CRP, adiponectin, TNF- $\alpha$, IL-6, ICAM-1, VCAM-1, FMD) in adults. It was hypothesised that the regular inclusion of nuts in a diet would improve markers of inflammation and endothelial function.

\section{METHODS}

This systematic review and meta-analysis followed the requirements of the Preferred Reporting Items for Systematic Reviews and Meta-Analyses statement ${ }^{25}$ (see online supplementary material 1 ). The review was registered in International Prospective Register of Systematic Reviews (PROSPERO) (http://www.crd.york.ac.uk/ PROSPERO; registration number: CRD42016045424).

\section{Study selection}

A systematic search of the databases MEDLINE, PubMed, Cumulative Index to Nursing and Allied Health Literature and Cochrane Central Register of Controlled Trials was conducted (all years to 13 January 2017). In line with recommendations by Rosen and Suhami, ${ }^{26}$ both MEDLINE and PubMed were searched to ensure recent studies were detected. Furthermore, where possible, Medical Subject Heading terms as well as free-text search terms were used in the search. ${ }^{26}$ Reference lists of eligible articles and relevant reviews were also reviewed for potential studies. An example of the search strategy used is shown in online supplementary material 2. Articles were restricted to those published in English.

To be included in this review, studies were required to meet the following inclusion criteria: (1) randomised controlled trial (including both parallel and crossover designs) or prospective cohort design; (2) studies conducted in humans aged 18 years or older; (3) studies assessing the effect of consumption of tree nuts or peanuts on an outcome of interest (CRP, adiponectin, TNF-alpha, IL-6, ICAM-1 VCAM-1, FMD), where the effect of nut consumption could be isolated. The outcomes of interest were selected to cover a suite of biomarkers regularly used in the literature to indicate changes to inflammation and endothelial dysfunction, including in previous meta-analyses exploring the effects of foods and dietary patterns ${ }^{2728}$; (4) studies with an intervention duration of 3 weeks or more (in the case of randomised controlled trials). This minimum duration was selected to ensure included studies reflected sustained changes to inflammation and endothelial function and to align with similar cut-offs used in other meta-analyses exploring the impact of dietary components on inflammation ${ }^{27}$ or the effect of nut consumption on other physiological measures. ${ }^{29}{ }^{30} \mathrm{In}$ addition, the following exclusion criteria were applied: (1) studies involving pregnant or breastfeeding women and (2) studies exploring the effects of nut oils or extracts.

Articles were screened based on title and abstract. Full texts were retrieved in the case that an abstract was not available or did not provide sufficient information to draw a conclusion regarding inclusion in the current review. In the case that results from one study were reported in multiple articles, all articles were checked to avoid duplication of study populations in the analysis or overlooking new information on outcomes. Where different information on outcomes were reported across articles, all relevant articles were included in line with the guidelines of the Cochrane Handbook. ${ }^{31}$ Where the same outcomes from a single study were reported across multiple articles, decisions relating to article inclusion were based first on the length of follow-up for the outcome and then by sample size.

\section{Data extraction}

The following data were extracted from each study: citation, country, sample size, participant age and body mass index, health status, study design, study duration, nut type, nut dose, details of control arm and background diet. Mean changes in relevant outcomes were extracted where possible, and in the case that these data were not available, mean final values were retrieved as recommended by the Cochrane Handbook for Systematic Reviews of Interventions. ${ }^{31}$ Study authors were contacted for additional details if the published article did not provide sufficient information. Where a study involved more than one intervention group meeting the inclusion criteria, data for the two intervention groups were combined as recommended by the Cochrane Handbook. ${ }^{31}$ In the case of the PREDIMED study, ${ }^{24}$ which included two intervention arms featuring a Mediterranean diet supplemented with either nuts or olive oil and a low fat control arm, data from the arm receiving the Mediterranean diet with olive oil was treated as the comparator group. This decision was 
made to ensure outcomes were not confounded by differences in the background diet of the two groups. Where studies reported median rather than mean, medians were used in the meta-analysis, and SD was imputed from IQR.

Abstract screening, study inclusion and exclusion and data extraction were conducted independently by two authors (EN and VG), and any disagreements were resolved via consensus.

\section{Statistical analyses}

Review Manager (RevMan V.5.3 (Copenhagen: The Nordic Cochrane Centre, the Cochrane Collaboration, 2014) was used to conduct random effects meta-analyses to determine the weighted mean differences (with $95 \%$ CIs) in change or final mean values for each outcome. In initial analyses, cross-over studies were treated in the same way as parallel studies by comparing measurements from the intervention periods with the control periods via a paired analysis, as the most conservative approach to managing cross-over studies. ${ }^{31}$ To explore whether this approach affected the final result by underweighting these studies, paired analyses of cross-over studies using correlation coefficients of $0.25,0.5$ and 0.75 were conducted as sensitivity analyses.

The proportion of total variation attributable to between-study heterogeneity was estimated using the $\mathrm{I}^{2}$ test statistic. ${ }^{32}$ An $\mathrm{I}^{2}$ value of $75 \%$ or greater was deemed to indicate a high level of inconsistency, based on the recommendations by Higgins et $a l^{32} \mathrm{I}^{2}$ values were generated for each analysis, including subgroup analyses (outlined below). For outcomes with 10 or more strata, funnel plots were generated to explore small study effects, with Egger's test used to determine the extent of funnel plot asymmetry. ${ }^{33}$ Where funnel plot asymmetry was detected, sensitivity analyses using the trim-and-fill method were conducted to explore potential publication bias. ${ }^{34}$ Egger's test and the trim-and-fill method were conducted using Stata V.15 (StataCorp, 2017). In addition to the correlation coefficient sensitivity analyses outlined previously, sensitivity analyses were also conducted to explore the effect of removing studies with imputed SD from analyses and of removing each individual study in meta-analyses ('leave-one-out' analysis). Prespecified subgroup analyses were also conducted, based on study duration (less than 3 months vs more than 3 months), risk of bias and nut type. For the purpose of subgroup analyses, studies which compared the effects of two types of nuts to a control ${ }^{35} 36$ were classified as 'mixed nut studies'. Post hoc subgroup analyses were conducted based on health status of participants, whether the energy value of nuts was substituted for other foods, study design (parallel vs cross-over) and nut dose $\left(<50 \mathrm{~g}\right.$ per day vs $\geq 50 \mathrm{~g}$ per day $\left.^{29}\right)$.

\section{Quality assessment}

The Cochrane Collaboration Risk of Bias tool ${ }^{31}$ was used to determine the risk of bias in included studies. EN and VG separately appraised the risk of bias and disagreements were resolved by discussion until consensus was reached.
The quality of the body of evidence was then determined using grading of recommendations assessment, development and evaluation (GRADE), ${ }^{37}$ which considers study design, risk of bias, inconsistency, indirectness, imprecision and other considerations such as publication bias. GRADEproGDT software V.2015 (GRADEpro (www. gradepro.org) McMaster University, 2014) was used to conduct the quality of evidence appraisal.

\section{RESULTS}

\section{Characteristics of included studies}

A total of 5200 articles were identified from the systematic search and review of relevant reference lists. After applying exclusion criteria, 36 articles describing 32 studies ( 34 strata in pooled analyses) were included in the systematic review and meta-analysis. The process of study inclusion and exclusion is shown in figure 1. Data access is available on request.

Characteristics of included studies are shown in table 1. All included studies were randomised controlled trials. Although prospective cohort study designs were also considered, no cohort studies met the overall inclusion criteria for the review. The most common reason was that the cohortstudies did notreport on the association between nut consumption and an outcome of interest. Fourteen studies had a parallel design ${ }^{13} 1516193538-50$ and 17 had a cross-over design. ${ }^{14171820-223651-60}$ One study ${ }^{61}$ combined a parallel and cross-over design, where participants were initially randomised to one of two parallel groups (energy adjusted or ad libitum diet). In this study, each group then took part in the cross-over part of the study consisting of a walnut included period and a walnut excluded period. Among all studies, duration ranged from 4 weeks to 5 years, although $20^{14} 151718212235364142474952-5658-60$ out of 32 studies $(63 \%)$ had a duration of less than 3 months. Studies were conducted in Spain, ${ }^{16} 1820363843-4753$ the USA, ${ }^{14} 172239414850525455585961$ Australia, ${ }^{49}{ }^{51}$ India, $^{19} 40$ Canada,${ }^{56}$ South Korea, ${ }^{15}$ China, ${ }^{21}$ Brazil,${ }^{42}$ South Africa, ${ }^{35}$ Iran, ${ }^{57}$ New Zealand ${ }^{13}$ and Germany. ${ }^{60}$ Studies included participants who were healthy, ${ }^{49}{ }^{52}$ had risk factors for chronic diseasesuchasoverweightorobesity, dyslipidaemia, hypertension or prediabetes, ${ }^{131718203640-4247505153555658-60}$ had type 2 diabetes mellitus, ${ }^{1421} 224857$ met the criteria for metabolic syndrome, ${ }^{1516193538}$ had diagnosed coronary artery disease $^{54}$ or included a mixture of the aforementioned conditions. ${ }^{3943-4661}$ Included studies examined the effects of consumption of a range of tree nuts including walnuts, ${ }^{17} 182239505253556061$ almonds, ${ }^{21} 4148545658$ pistachios, ${ }^{14} 1920405759$ hazelnuts, ${ }^{1347}$ mixed nuts ${ }^{15} 163843-46$ and Brazil nuts, ${ }^{49}$ as well as peanuts. ${ }^{4251}$ In addition, two studies included multiple intervention arms, featuring a different type of nut in each (walnuts and cashews, ${ }^{35}$ and walnuts and almonds ${ }^{36}$ ) compared with a control arm. Nuts were consumed in either prescribed doses, ranging from approximately $18^{49}$ to $85 \mathrm{~g}$ per day ${ }^{54}$ or were designed to provide a set proportion of dietary energy, so the amount would vary for individuals. ${ }^{1418192135505859}$ Background 


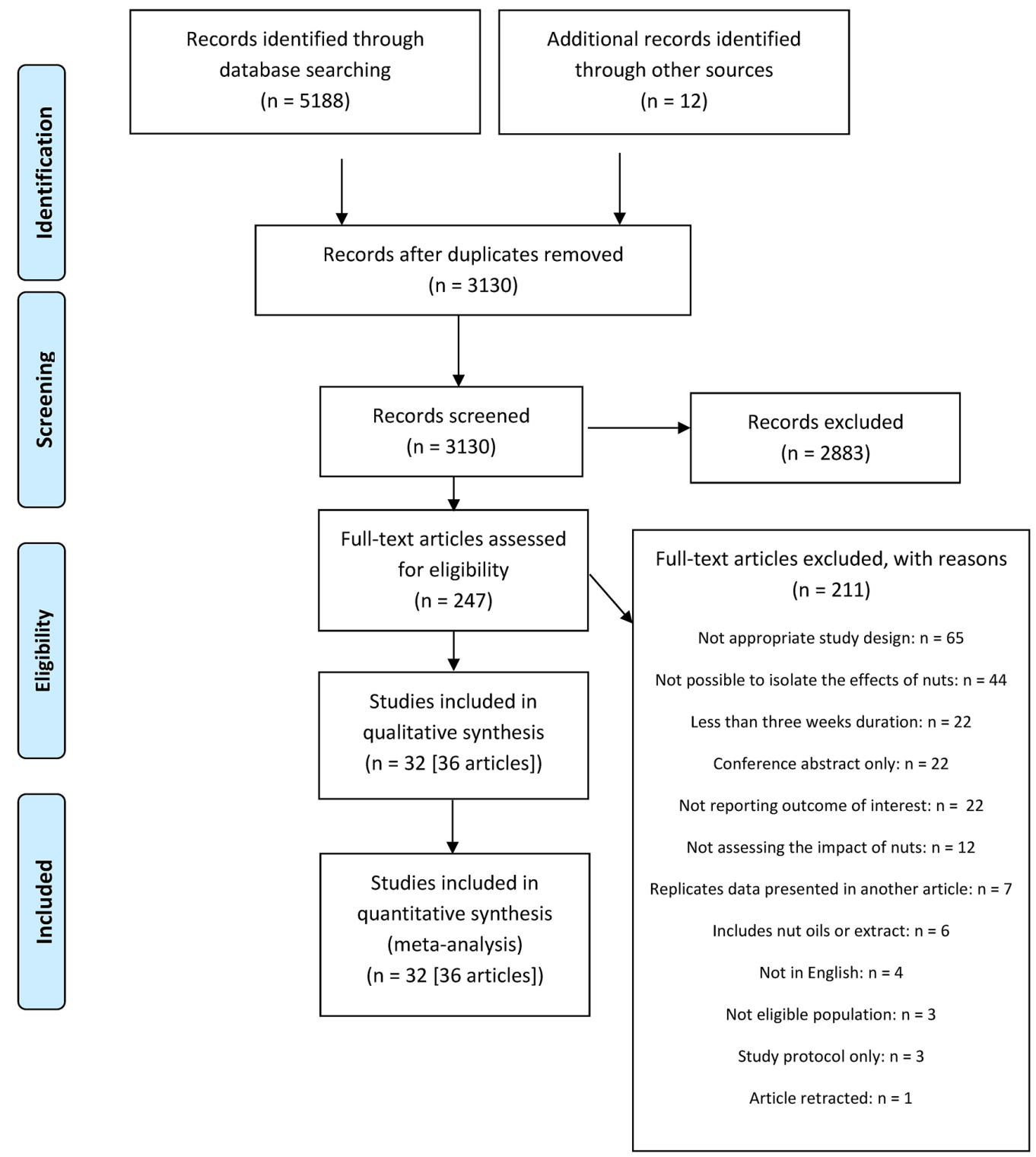

Figure 1 PRISMA ${ }^{25}$ flow diagram of study selection. PRISMA, Preferred Reporting Items for Systematic Reviews and MetaAnalyses.

diets consisted of either participant's habitual diet or a prescribed diet aligned with healthy lifestyles such as the National Cholesterol Education Program (NCEP) Step I or II diet, a Mediterranean-style diet, the Therapeutic Lifestyle Changes diet or another prudent style diet in line with dietary guidelines. Six studies provided all or the majority of foods under controlled feeding conditions. ${ }^{14} 2135555859$ Twenty-two studies ${ }^{1417-223536394042-475053-5658-60}$ prescribed diets accounting for the energy value of the nuts, either quantitatively through dietary modelling (including the energy value of the nuts within the total energy value of the diet) or qualitatively by encouraging participants to substitute nuts for items with similar energy values. One study $^{61}$ included an intervention group where participants were advised on food substitutions to account for the energy value of the provided nuts and another intervention group where energy intake was not prescribed (ad libitum food consumption). During the control diets or periods, participants typically consumed a similar diet but without nuts, although some studies included control diets with a specific product substituted for the nuts, such as eggs, ${ }^{52}$ olive oil,${ }^{3643-46}$ muffins $^{56}$ and chocolate, ${ }^{41}$ among others. Only two studies ${ }^{4250}$ stated they prescribed a set energy restriction for both intervention and control groups; all other studies used isocaloric diets for weight maintenance or ad libitum diets. No studies reported a significant difference in weight loss between the intervention and control groups.

\section{Effect of nut consumption on study outcomes}

Flow-mediated dilation

A total of nine strata from eight studies ${ }^{14} 17182240545961$ explored the effect of nut consumption on FMD. Of the nine strata, five explored the effect of walnut consumption on FMD, ${ }^{17} 182261$ and six had a duration of less than 3 months. ${ }^{141718225459}$ The meta-analysis showed that nut 

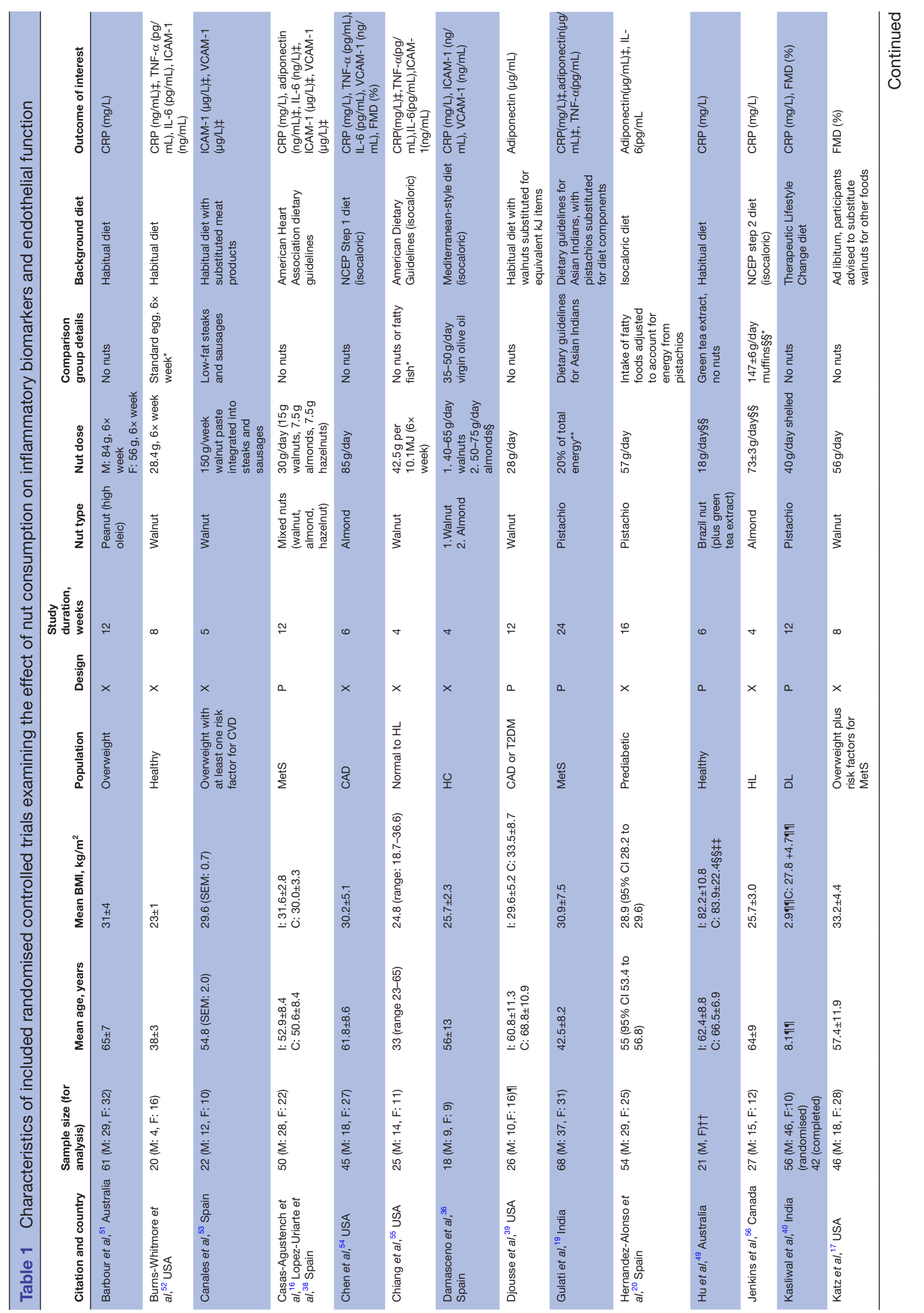

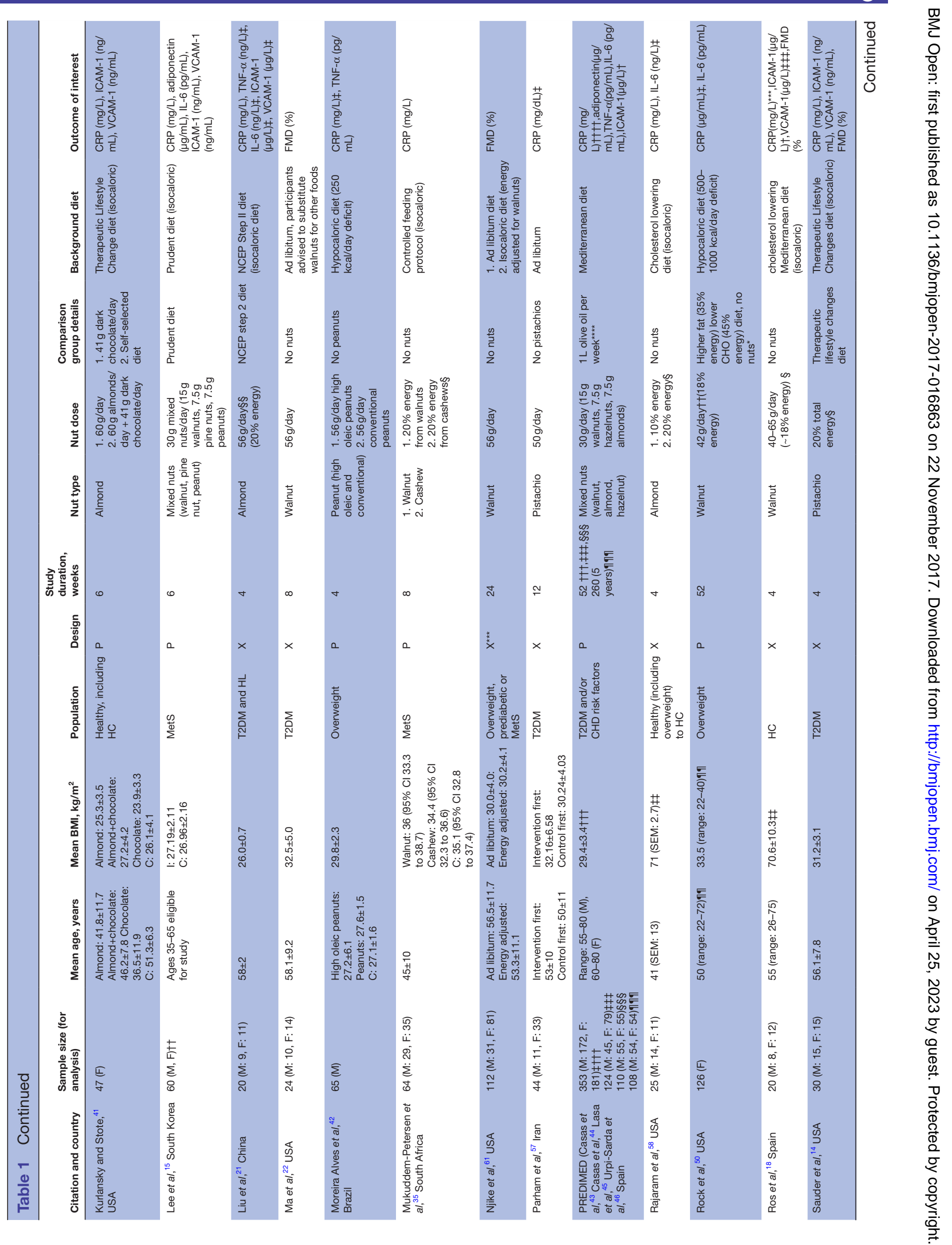


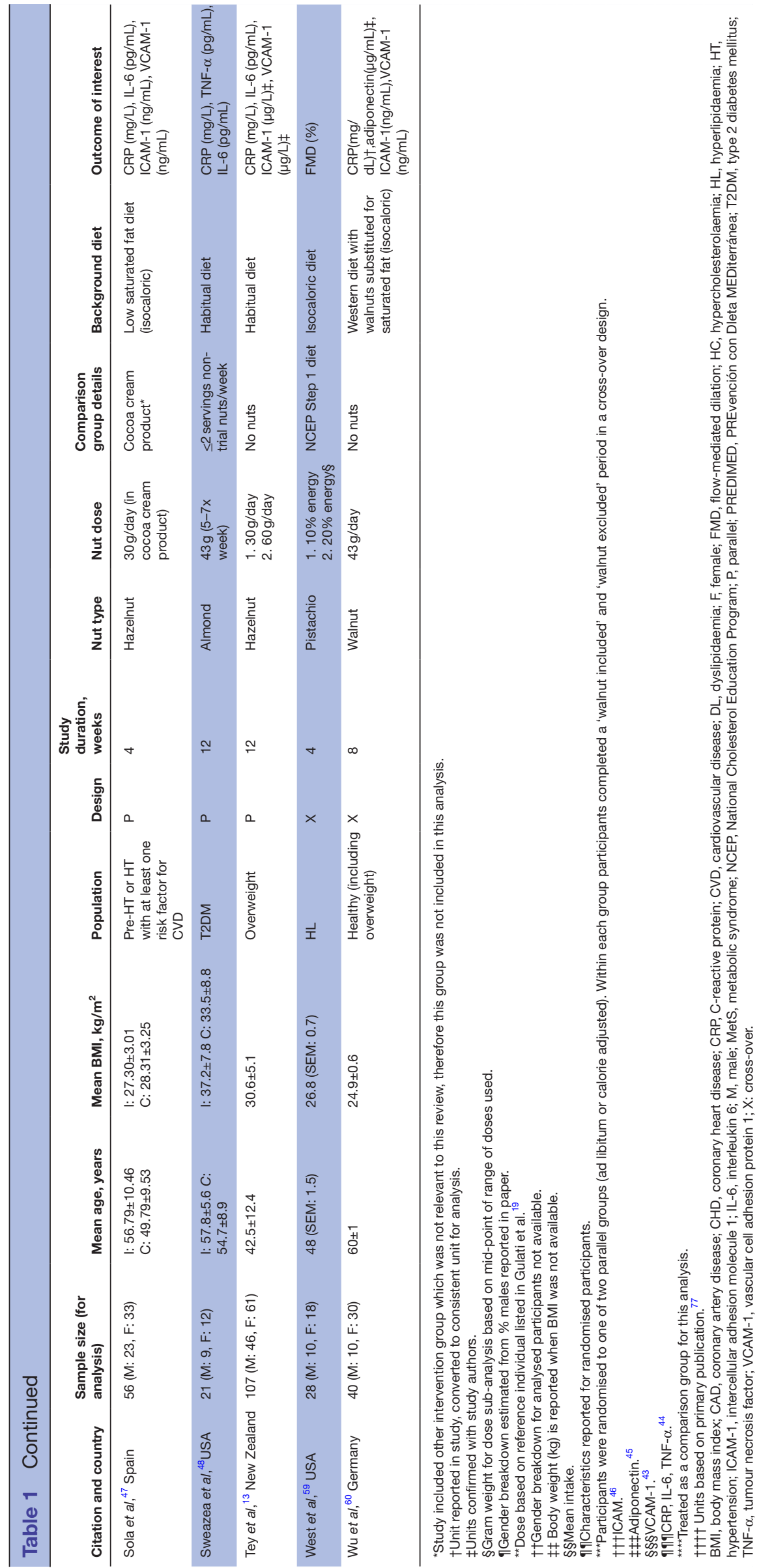




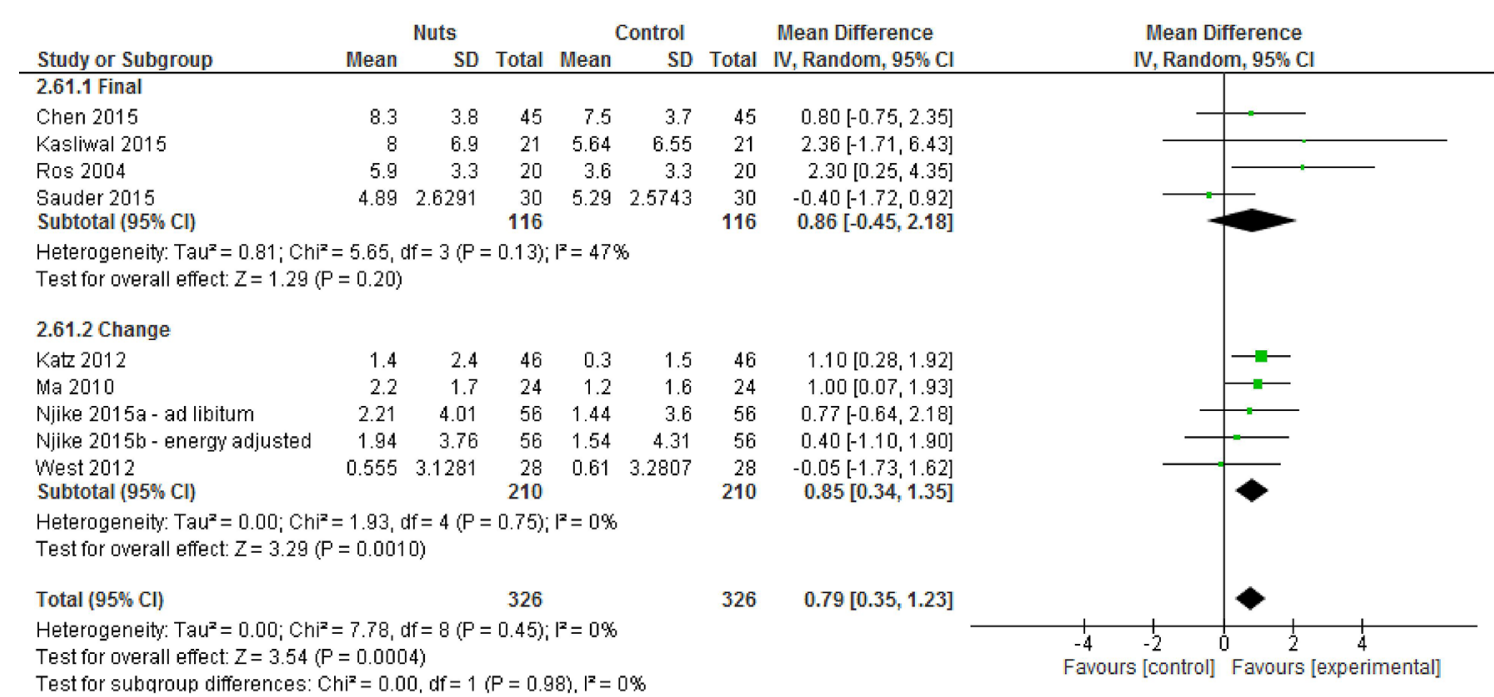

Figure 2 Difference in FMD (\%) between nut consumption and control (presented as subgroups based on mean final or change values for readability). Diamond indicates weighted mean difference with 95\% Cls. FMD, flow-mediated dilation.

consumption was associated with a significant increase in FMD (figure 2 and table 2). Sensitivity analyses indicated that excluding any one study did not substantially alter the effect (data not shown). The effect estimate was also similar after using different correlation coefficients (CC: 0.5 ; see online supplementary material 3; CC: 0.25 and 0.75 , data not shown). No significant differences were found for subgroup analyses (see online supplementary material 4) although it was noted that when subgroup comparisons were made according to nut type, only the walnut subgroup found significant improvements in FMD.

\section{C-reactive protein}

Atotalof26stratafrom 25studies ${ }^{13-16181921353640-424447-5254-5860}$ explored the effect of nut consumption on CRP. Almonds were the most common nut type used in these analyses (seven strata ${ }^{214148545658}$ ), followed by walnuts ${ }^{1850525560}$ and mixtures of more than one nut type $\mathrm{e}^{1516353644}$ (each used in five strata). A total of 17 strata from 16 studies had a duration of less than 3 months. ${ }^{1415} 18213536414247495254-565860$ When all studies were included in the meta-analysis, nut consumption resulted in non-significant differences in CRP (figure 3 and table 2). The overall effect was relatively unchanged when studies with imputed SD were removed from the analysis (table 2). Sensitivity analyses identified two studies ${ }^{15} 52$ that contributed substantially to the pooled result, as when they were excluded from the meta-analysis, the reductions in CRP were significant (see online supplementary material 5). In addition, the use of different correlation coefficients did not change the overall effect found (CC: 0.5 , see online supplementary material 3; CC: 0.25 and 0.75 , data not shown). Subgroup analyses indicated that statistically significant differences were found between studies which included the energy value of nuts in the prescribed diet compared with those that did not (see online supplementary material 4 ). An effect estimate of $-0.23 \mathrm{mg} / \mathrm{L}(-0.44$ to -0.01$)$ was found for studies in which diets incorporated the energy value of nuts, while an effect estimate of $-0.00 \mathrm{mg} / \mathrm{L}(-0.06$ to $0.05)$ ) was found for studies which did not incorporate the energy value of nuts $\left(\chi^{2}=3.99, \mathrm{df}=1(\mathrm{P}=0.05), \mathrm{I}^{2}=74.9 \%\right)$. When studies were grouped according to nut dose, an effect estimate of $-0.00 \mathrm{mg} / \mathrm{L}(0.00$ to 0.00$)$ was found for studies which included less than $50 \mathrm{~g}$ of nuts/day, while an effect estimate of $-0.34 \mathrm{mg} / \mathrm{L}(-0.63$ to-0.06) was found when $50 \mathrm{~g}$ or more were used $\left(\chi^{2}=5.74, \mathrm{df}=1\right.$ $\left.(\mathrm{P}=0.02), \mathrm{I}^{2}=82.6 \%\right)$. Borderline significant differences $(\mathrm{P}=0.05)$ were found when studies with a parallel design were compared with cross-over studies. However, when either of the studies identified in the sensitivity analysis ${ }^{152}$ were excluded, these subgroup analyses no longer produced significant results (data not shown).

\section{Adiponectin, TNF- $\alpha$, IL-6, ICAM-1, VCAM-1}

The meta-analysis showed that consumption of nuts did not result in significant differences in adiponectin, TNF- $\alpha$, IL-6, ICAM-1 or VCAM-1 (table 2 and online supplementary material 6 ). In the case that pooled analyses featured studies with imputed SDs (IL-6, ICAM-1, VCAM-1), excluding these studies did not substantially change the effect estimates (table 2). Sensitivity analyses indicated that excluding any one study did not substantially alter the effect (data not shown). Overall effects also did not change when different correlation coefficients were used for cross-over studies (CC: 0.5, see online supplementary material 3; CC: 0.25 and 0.75 , data not shown). No significant differences between subgroups were observed (see online supplementary material 4).

\section{Small study effects}

Funnel plots were generated for outcomes with 10 or more strata (CRP, IL-6, ICAM-1 and VCAM-1) (see online supplementary material 7). Egger's test indicated asymmetry in funnel plots for CRP (bias $=-0.68(95 \%$ $\mathrm{CI}=-1.06$ to -0.31$), \mathrm{P}=0.001)$ and $\mathrm{IL}-6$ (bias $=-0.81(95 \%$ 


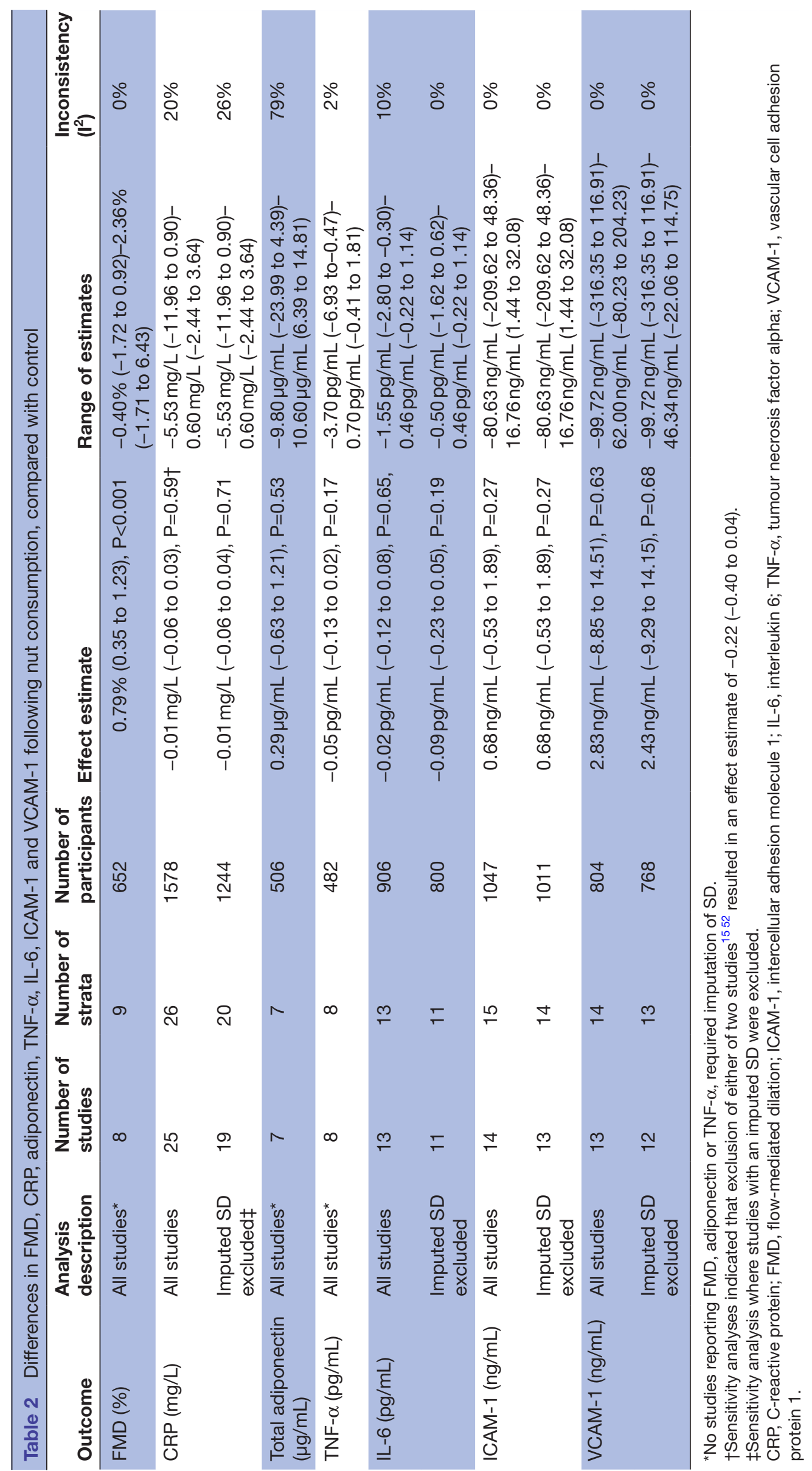




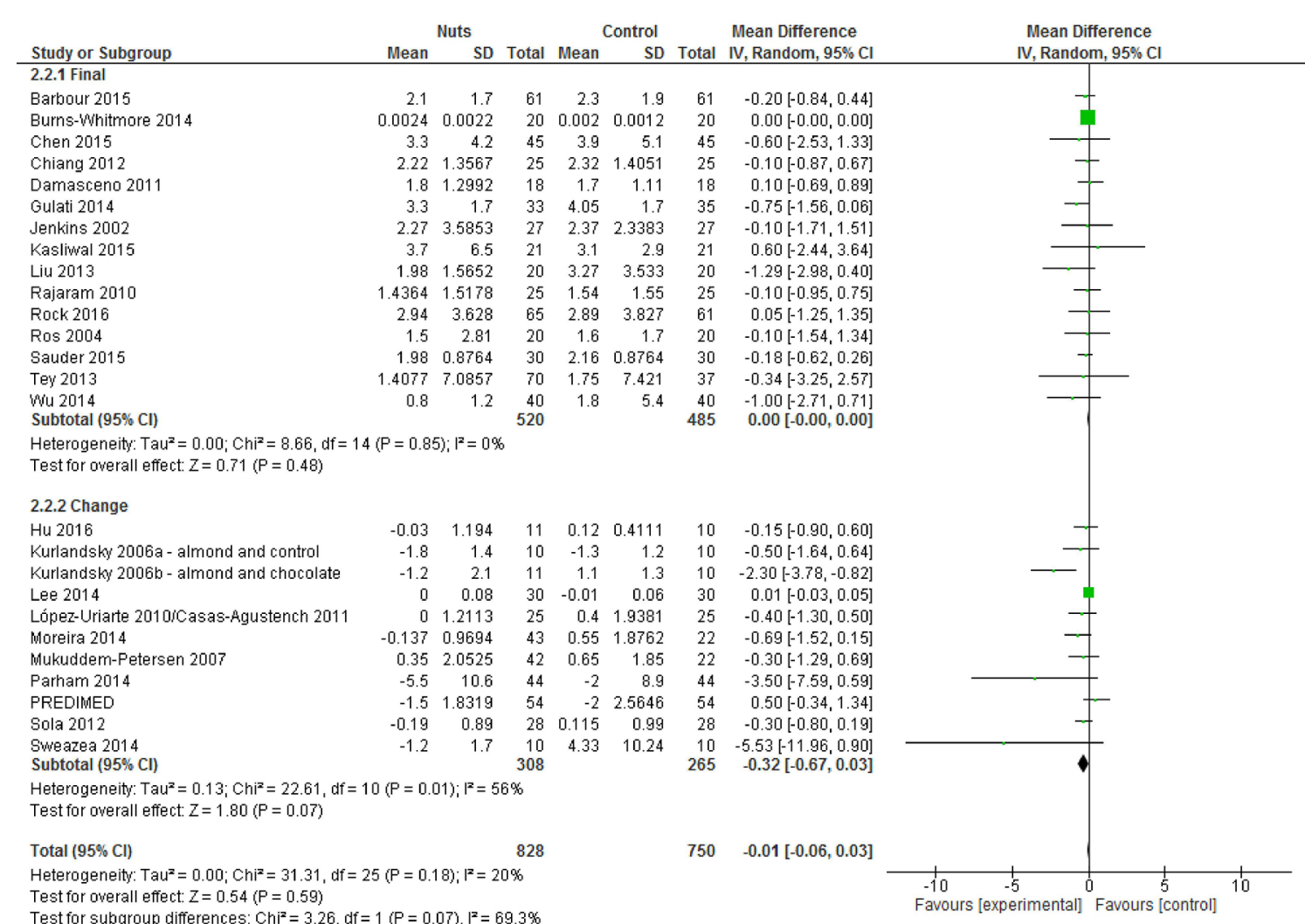

Figure 3 Difference in C-reactive protein $(\mathrm{mg} / \mathrm{L}$ ) between nut consumption and control (presented as subgroups based on mean final or change values for readability). Diamond indicates weighted mean difference with $95 \% \mathrm{Cls}$.

$\mathrm{CI}=-1.45$ to -0.16$), \mathrm{P}=0.02)$, suggesting the presence of small study effects which may have been attributable to publication bias. Use of the trim-and-fill method did not change these results (data not shown). Funnel plot asymmetry was not detected for ICAM-1 or VCAM-1 (data not shown).

\section{Risk of bias and quality of the body of evidence}

The risk of bias was determined for each strata using the Cochrane Risk of Bias Tool, and the results of the assessment are shown in figure 4 and online supplementary materials 8 and 9. The quality of the evidence was 'high' for FMD, ICAM-1 and VCAM-1. The quality was downgraded to 'moderate' for TNF- $\alpha$ due to risk of bias and to 'low' for CRP and IL-6 due to both risk of bias and the possibility of publication bias. The quality of the evidence for adiponectin was downgraded to 'very low' due to risk of bias, inconsistency and imprecision (see online supplementary material 10).

\section{DISCUSSION}

The results of this systematic review and meta-analysis suggested favourable effects of nut consumption on FMD, a measure of endothelial function. These findings align with a review conducted in 2011 by the European Food Safety Authority (EFSA), which explored the effects of walnut consumption on endothelium-dependent vasodilation. ${ }^{62} \mathrm{~A}$ meta-analysis was not part of the EFSA

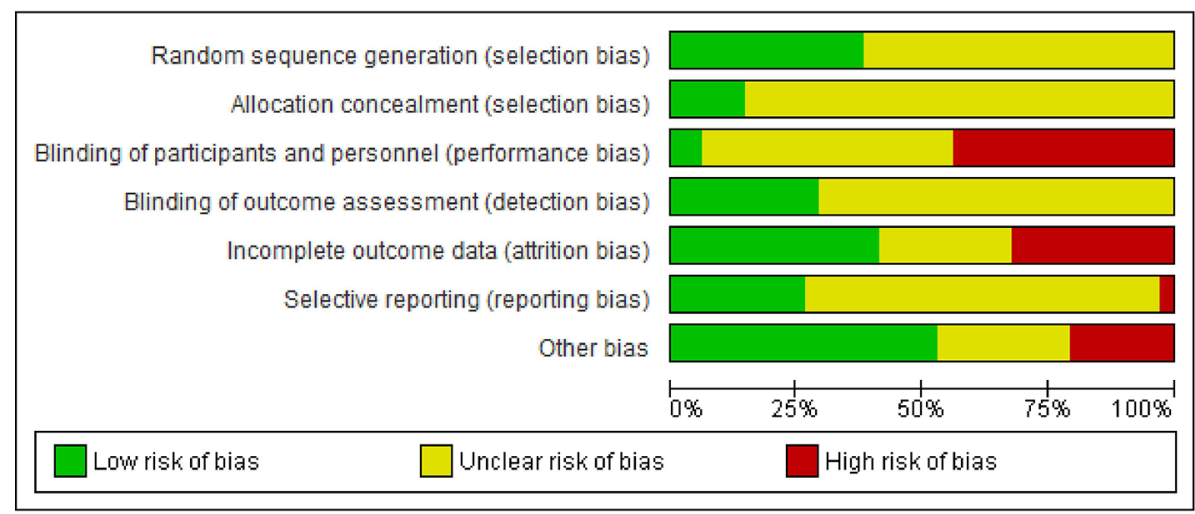

Figure 4 Risk of bias assessment as proportion of total strata. 
report, ${ }^{62}$ but the present study provides a meta-analysis that includes more recently published research. ${ }^{1761}$ It also includes studies investigating other types of nuts. ${ }^{14} 405459$ Subgroup analyses found significant improvements in FMD only in those studies using walnuts, consistent with the EFSA report which only examined walnut consumption, although the test for subgroup differences in the present study did not reach statistical significance. This may have resulted from the small number of studies available for assessing FMD. Having few studies may have also played a role in the lack of significant effects observed in other FMD subgroup analyses. These include studies in participants with type 2 diabetes or studies lasting longer than 3 months. Further research is therefore required in this area.

Despite the small sample size, the findings of this review relating to FMD are of value due to the known associations between FMD and future cardiovascular events. A meta-analysis of cohort studies found a significant reduction in risk of cardiovascular events per $1 \%$ increase in FMD (relative risk: 0.872 (95\% CI 0.832 to 0.914$)){ }^{8}$ In comparison, the present study found an effect estimate of $0.79 \%$ for nut consumption compared with controls, suggesting these results are likely to be of clinical relevance to future cardiovascular risk. There are a number of mechanisms by which nuts, and walnuts in particular, could improve FMD. FMD is a measure of endothelial dysfunction, ${ }^{63}$ a condition characterised by reduced availability of the vasodilator nitric oxide $(\mathrm{NO}) .{ }^{64}$ Nuts contain high levels of L-arginine, ${ }^{65}$ an amino acid which acts as a precursor to NO. ${ }^{66}$ Walnuts in particular are rich in alpha-linolenic acid, a polyunsaturated fatty acid that has been suggested to increase membrane fluidity, thus also increasing nitric oxide synthesis and release. ${ }^{67}$ The antioxidant content of nuts may also play a role in the improvements in endothelial function observed. ${ }^{9}$

Our finding of no significant effects on inflammatory biomarkers CRP, TNF- $\alpha$, IL-6, ICAM-1, VCAM-1 or the anti-inflammatory biomarker adiponectin reflects the body of evidence available at this time. There may be effects with CRP but characteristics of the study sample or design of the dietary intervention may influence the ability to detect these effects. Sensitivity analyses indicated that results may have been disproportionally influenced by a small number of studies. Exclusion of either one of two studies ${ }^{15} 52$ resulted in the meta-analysis yielding significant reductions in CRP following nut intake, suggesting these two studies were responsible for the results found. This appears to be the result of low reported CRP values and correspondingly small standard errors, resulting in these studies receiving substantially higher weighting than other studies in the pooled analysis. The study sample may in part explain these findings, as the study by Burns-Whitmore et $a \tilde{l}^{2}$ was conducted in healthy lacto-ovo vegetarians. Consumption of a plantbased diet has been associated with decreased inflammation. ${ }^{68}$ In contrast, Lee $e t a l^{15}$ explored the effect of nut consumption in individuals with metabolic syndrome, which is typically associated with elevated CRP levels. ${ }^{69}$ Reported units were confirmed with study authors.

The findings of this review may also have been influenced by the design of the dietary interventions included. Subgroup analyses found significant reductions in CRP when studies incorporated $50 \mathrm{~g}$ or more of nuts per day. This finding aligns with previous research suggesting a dose-response effect of nut intake on other outcomes such as cholesterol. ${ }^{70}$ However, these findings should be interpreted with caution, as several studies ${ }^{14} 18192135505859$ incorporated nuts as a proportion of total energy, resulting in substantial variation between individuals in the dose consumed. Furthermore, whether the energy value of nuts was adjusted for in the total diet may have influenced results. Subgroup analyses suggested significant effects on CRP were only found when the energy provided by nuts was accounted for either by dietary modelling or advice to substitute other foods for nuts. This aligns with a previous review by our group which highlighted the importance of considering total energy intake in trials examining the effect of vegetable intake on weight loss. ${ }^{71}$ There is also evidence to suggest markers of inflammation such as CRP may be reduced following periods of energy restriction, ${ }^{72}$ highlighting the importance of considering total energy intake when exploring the effects of individual foods. The design of the control arm may have also impacted on results, as several studies ${ }^{3643-46}$ compared intake of nuts with a control intervention which also had the potential to influence inflammation and endothelial function, for example olive oil. ${ }^{27}$ The potential impact of control groups on underestimating intervention effects has previously been highlighted in the weight loss literature. ${ }^{73}$ Trials aiming to explore the influence of specific foods on health outcomes must carefully consider the design of the dietary intervention and control arms and avoid increases in total energy intake which could skew results.

The heterogeneity in study design elements, particularly related to dietary intervention, may explain why reviews exploring the effects of nut consumption on inflammation have found varying results. Although including fewer studies than in our review, a recently published review by Mazidi et $a l^{23}$ also found non-significant differences in inflammatory biomarkers (CRP, IL-6, adiponectin, ICAM-1 and VCAM-1), although in contrast to our review, they observed a small increase in CRP levels. The review by Mazidi $e t^{23}$ appeared to have broader eligibility criteria which also included postprandial studies and those exploring the effects of soy consumption. In another review, Barbour $e t a l^{74}$ reported significant reductions in CRP following nut consumption. It should be noted however, that Barbour $e t a l^{74}$ included studies where nut consumption was encouraged as part of a suite of favourable dietary changes not matched in control groups, which means that the effect of the nuts themselves could not be isolated. In these circumstances, it may not be possible to show whether effects observed were the result of increases in nut intake or the wider dietary changes occurring. We avoided this problem by 
excluding studies with a portfolio of dietary changes not matched in the control group or by treating a comparable intervention group as the 'control' (or comparator), as in the case of the PREDIMED study. ${ }^{24}$ Nevertheless, nuts appear in healthy dietary patterns, and we have previously shown that consumption of a healthy dietary pattern (many of which include habitual nut intake) results in significant reductions in CRP. ${ }^{75}$

It should be noted that while the current analysis found favourable effects of nut consumption on a marker of endothelial dysfunction, the lack of evidence for effects on cell adhesion molecules VCAM-1 and ICAM-1 suggests changes in endothelial cell activation may not have occurred. Given that the inflammatory cytokines which characteristically induce endothelial cell activation (for example TNF- $\alpha$ and IL- 6$)^{64}$ also appeared unchanged, the lack of difference found for ICAM- 1 and VCAM- 1 is perhaps not surprising. More research on this cluster of molecules will be informative.

This review had a number of strengths. It used a systematic methodology following current guidelines for systematic reviews, including prospective registration and used the Cochrane Risk of Bias tool and GRADE method to evaluate the quality of evidence. We considered a range of biomarkers associated with inflammation and endothelial function, including the anti-inflammatory adipocyte adiponectin. These biomarkers were selected to reflect changes in disease progression and amelioration to explore mechanisms responsible for the favourable effects of nut consumption on cardiovascular disease $^{10}$ and other chronic conditions. ${ }^{11}{ }^{12}$ However, we fully acknowledge that the measures explored here are not interchangeable with disease endpoints such as mortality and morbidity. The size of the evidence base, including the small number of participants available for analyses of individual biomarkers, is a limitation, particularly with respect to generalisability and strength of the evidence. Furthermore, although we were unable to explore the distribution of the published data included in this meta-analysis, the fact that several studies reported median values rather than means suggests some of the data may have been skewed, which may have impacted on our analyses.

The heterogeneity of the evidence base included can be also considered a limitation of this review. Variation existed as a result of participant health status, nut type and dose and study duration, although these factors were explored in subgroup analyses. Statistically significant subgroup differences were found only for CRP when studies were grouped according to whether they incorporated the energy value of nuts into the diet and based on nut dose $(<50 \mathrm{~g} /$ day vs $\geq 50 \mathrm{~g} /$ day $)$. However, due to the small number of studies, it is possible that other subgroup differences may have been found if the sample size was larger. For example, borderline significant differences $(\mathrm{P}=0.05)$ were found between the study designs, with larger reductions in CRP found for crossover design studies. As the nature of cross-over studies eliminates between-subject variation, ${ }^{76}$ they may provide superior insights when exploring the impact of dietary interventions on biomarkers such as CRP; however, their results may also be impacted by carry-over effects. ${ }^{31}$ Given the short or absent wash-out periods of some of the included studies, ${ }^{18} 36515558$ the potential impact of carry-over effects cannot be ruled out. Background diets also varied between studies, with some studies prescribing diets based on dietary guidelines, whereas others allowed participants to follow their habitual diet, which may have varied substantially between individuals. Analysis of funnel plots suggested the results for CRP and IL-6 may have been influenced by small study effects (which could indicate publication bias), which resulted in downgrading the quality of the evidence for these outcomes. Funnel plot asymmetry remained after sensitivity analyses were conducted. These findings suggest the need for more research in this area, with a particular focus on the registration of study protocols with detailed information on primary and secondary outcomes, to reduce the potential for publication bias.

This systematic review and meta-analysis of the effects of nut consumption on inflammation and endothelial function found evidence for favourable effects on FMD, a measure of endothelial function. Non-significant differences in CRP, adiponectin, TNF- $\alpha$, IL-6, ICAM-1 and VCAM-1 suggest a lack of consistent available evidence for effects of nut consumption on inflammation, although the results for CRP should be interpreted with caution due to the large influence of single studies on the pooled results. The findings of this review provide further insight into the mechanisms by which nut consumption may exert favourable effects on the risk of chronic conditions such as cardiovascular disease. The findings also build on previous research such as the 2011 EFSA report ${ }^{62}$ on walnut consumption and endothelial-dependent vasodilation and reinforce the value of including nuts within a healthy dietary pattern. However, the small evidence base for FMD and the observed lack of consistency in findings relating to inflammation suggest a need for more research in this area, with a particular focus on randomised controlled trials incorporating the energy value of nuts into the total diet. There is also a need for the transparent registration of trial protocols, as well as appropriate dietary controls. These could include healthy dietary patterns (not including nuts), with a greater emphasis on dietary modelling required to ensure nutrient intakes are matched between control and intervention groups, minimising the risk of confounding.

Contributors Study concept and design: EPN, LCT, MJB. Acquisition, analysis or interpretation of data: all authors. Drafting of the manuscript: EPN. Critical revision of the manuscript for important intellectual content: all authors. Statistical analysis: EPN, VG, MJB. Obtained funding: LCT, EPN, MJB. Administrative, technical or material support: all authors. Study supervision: LCT and MJB.

Funding This study was supported by the INC International Nut and Dried Fruit Council (2016-R02) 
Disclaimer The funders approved the study design but had no other role in the collection, management, analysis and interpretation of the data or preparation of the manuscript for submission.

Competing interests EPN reports personal fees from Safcol Australia, personal fees from Nuts for Life, grants from Pork Cooperative Research Centre, grants from Australian Government Department of Health, outside the submitted work. LCT reports grants from Illawarra Health and Medical Research Institute, grants from California Walnut Commission, grants from Nuts for Life; personal fees from McCormicks Science Institute, non-financial support from California Walnut Commission, outside the submitted work.

Provenance and peer review Not commissioned; externally peer reviewed. Data sharing statement Access to data available on request (elizan@uow.edu. au).

Open Access This is an Open Access article distributed in accordance with the Creative Commons Attribution Non Commercial (CC BY-NC 4.0) license, which permits others to distribute, remix, adapt, build upon this work non-commercially, and license their derivative works on different terms, provided the original work is properly cited and the use is non-commercial. See: http://creativecommons.org/ licenses/by-nc/4.0/

(c) Article author(s) (or their employer(s) unless otherwise stated in the text of the article) 2017. All rights reserved. No commercial use is permitted unless otherwise expressly granted.

\section{REFERENCES}

1. Esser N, Legrand-Poels S, Piette J, et al. Inflammation as a link between obesity, metabolic syndrome and type 2 diabetes. Diabetes Res Clin Pract 2014;105:141-50.

2. Libby P, Ridker PM, Maseri A, et al. Inflammation and atherosclerosis. Circulation 2002;105:1135-43.

3. Silva D, Pais de Lacerda A. [High-sensitivity C-reactive protein as a biomarker of risk in coronary artery disease]. Rev Port Cardiol 2012;31:733-45.

4. Ruan H, Lodish HF. Insulin resistance in adipose tissue: direct and indirect effects of tumor necrosis factor-alpha. Cytokine Growth Factor Rev 2003;14:447-55.

5. Gabay C. Interleukin-6 and chronic inflammation. Arthritis Res Ther 2006;8 Suppl 2:S3

6. Stoner L, Lucero AA, Palmer BR, et al. Inflammatory biomarkers for predicting cardiovascular disease. Clin Biochem 2013;46:1353-71.

7. Robinson K, Prins J, Venkatesh B. Clinical review: adiponectin biology and its role in inflammation and critical illness. Crit Care 2011;15:221.

8. Inaba Y, Chen JA, Bergmann SR. Prediction of future cardiovascular outcomes by flow-mediated vasodilatation of brachial artery: a metaanalysis. Int J Cardiovasc Imaging 2010;26:631-40.

9. Casas-Agustench P, Bulló M, Salas-Salvadó J. Nuts, inflammation and insulin resistance. Asia Pac J Clin Nutr 2010;19:124-30.

10. Luo $C$, Zhang $Y$, Ding $Y$, et al. Nut consumption and risk of type 2 diabetes, cardiovascular disease, and all-cause mortality: a systematic review and meta-analysis. Am J Clin Nutr 2014;100:256-69.

11. Ibarrola-Jurado N, Bulló M, Guasch-Ferré M, et al. Cross-sectional assessment of nut consumption and obesity, metabolic syndrome and other cardiometabolic risk factors: the PREDIMED study. PLoS One 2013;8:e57367.

12. Afshin A, Micha R, Khatibzadeh S, et al. Consumption of nuts and legumes and risk of incident ischemic heart disease, stroke, and diabetes: a systematic review and meta-analysis. Am J Clin Nutr 2014;100:278-88.

13. Tey SL, Gray AR, Chisholm AW, et al. The dose of hazelnuts influences acceptance and diet quality but not inflammatory markers and body composition in overweight and obese individuals. J Nutr 2013:143:1254-62

14. Sauder KA, McCrea CE, Ulbrecht JS, et al. Effects of pistachios on the lipid/lipoprotein profile, glycemic control, inflammation, and endothelial function in type 2 diabetes: A randomized trial. Metabolism 2015;64:1521-9.

15. Lee YJ, Nam GE, Seo JA, et al. Nut consumption has favorable effects on lipid profiles of Korean women with metabolic syndrome. Nutr Res 2014;34:814-20.

16. Casas-Agustench $P$, López-Uriarte $P$, Bulló $M$, et al. Effects of one serving of mixed nuts on serum lipids, insulin resistance and inflammatory markers in patients with the metabolic syndrome. Nutr Metab Cardiovasc Dis 2011;21:126-35.
17. Katz DL, Davidhi A, Ma Y, et al. Effects of walnuts on endothelial function in overweight adults with visceral obesity: a randomized, controlled, crossover trial. J Am Coll Nutr 2012;31:415-23.

18. Ros E, Núñez I, Pérez-Heras A, et al. A walnut diet improves endothelial function in hypercholesterolemic subjects: a randomized crossover trial. Circulation 2004;109:1609-14.

19. Gulati S, Misra A, Pandey RM, et al. Effects of pistachio nuts on body composition, metabolic, inflammatory and oxidative stress parameters in Asian Indians with metabolic syndrome: a 24-wk, randomized control trial. Nutrition 2014;30:192-7.

20. Hernández-Alonso P, Salas-Salvadó J, Baldrich-Mora M, et al. Beneficial effect of pistachio consumption on glucose metabolism, insulin resistance, inflammation, and related metabolic risk markers: a randomized clinical trial. Diabetes Care 2014;37:3098-105.

21. Liu JF, Liu YH, Chen CM, et al. The effect of almonds on inflammation and oxidative stress in Chinese patients with type 2 diabetes mellitus: a randomized crossover controlled feeding trial. Eur J Nutr 2013:52:927-35.

22. Ma Y, Njike VY, Millet J, et al. Effects of walnut consumption on endothelial function in type 2 diabetic subjects: a randomized controlled crossover trial. Diabetes Care 2010;33:227-32.

23. Mazidi M, Rezaie P, Ferns GA, et al. Impact of different types of tree nut, peanut, and soy nut consumption on serum C-reactive protein (CRP): A systematic review and meta-analysis of randomized controlled clinical trials. Medicine 2016;95:e5165.

24. Estruch R, Ros E, Salas-Salvadó J, et al. Primary prevention of cardiovascular disease with a Mediterranean diet. $N$ Engl J Med 2013;368:1279-90.

25. Moher D, Liberati A, Tetzlaff J, et al. Preferred reporting items for systematic reviews and meta-analyses: the PRISMA statement. PLoS Med 2009;6:e1000097.

26. Rosen L, Suhami R. The art and science of study identification: a comparative analysis of two systematic reviews. BMC Med Res Methodol 2016;16:24.

27. Schwingshackl L, Christoph M, Hoffmann G. Effects of olive oil on markers of inflammation and endothelial function: a systematic review and meta-analysis. Nutrients 2015;7:7651-75.

28. Schwingshackl L, Hoffmann G. Mediterranean dietary pattern, inflammation and endothelial function: a systematic review and meta-analysis of intervention trials. Nutr Metab Cardiovasc Dis 2014;24:929-39.

29. Blanco Mejia S, Kendall CW, Viguiliouk E, et al. Effect of tree nuts on metabolic syndrome criteria: a systematic review and meta-analysis of randomised controlled trials. BMJ Open 2014;4:e004660.

30. Viguiliouk E, Kendall CW, Blanco Mejia S, et al. Effect of tree nuts on glycemic control in diabetes: a systematic review and meta-analysis of randomized controlled dietary trials. PLoS One 2014;9:e103376.

31. Higgins JPT, Green S. Cochrane Handbook for Systematic Reviews of Interventions Version 5.1.0 [updated March 2011]. The Cochrane Collaboration 2011.

32. Higgins JP, Thompson SG, Deeks JJ, et al. Measuring inconsistency in meta-analyses. BMJ 2003;327:557-60.

33. Egger M, Davey Smith G, Schneider M, et al. Bias in meta-analysis detected by a simple, graphical test. BMJ 1997;315:629-34.

34. Duval S, Tweedie R. Trim and fill: A simple funnel-plot-based method of testing and adjusting for publication bias in meta-analysis. Biometrics 2000;56:455-63.

35. Mukuddem-Petersen J, Stonehouse Oosthuizen W, Jerling JC, et al. Effects of a high walnut and high cashew nut diet on selected markers of the metabolic syndrome: a controlled feeding trial. $\mathrm{Br} \mathrm{J}$ Nutr 2007;97:1144.

36. Damasceno NR, Pérez-Heras A, Serra M, et al. Crossover study of diets enriched with virgin olive oil, walnuts or almonds. Effects on lipids and other cardiovascular risk markers. Nutr Metab Cardiovasc Dis 2011;21:S14-20.

37. Guyatt $\mathrm{GH}$, Oxman $\mathrm{AD}$, Vist GE, et al; GRADE: an emerging consensus on rating quality of evidence and strength of recommendations, 2008

38. López-Uriarte P, Nogués R, Saez G, et al. Effect of nut consumption on oxidative stress and the endothelial function in metabolic syndrome. Clin Nutr 2010;29:373-80.

39. Djoussé L, Lu B, Gaziano JM. Effects of walnut consumption on endothelial function in people with type 2 diabetes: a randomized pilot trial. Curr Nutr Rep 2016;5:1-8.

40. Kasliwal RR, Bansal M, Mehrotra R, et al. Effect of pistachio nut consumption on endothelial function and arterial stiffness. Nutrition 2015;31:678-85

41. Kurlandsky SB, Stote KS. Cardioprotective effects of chocolate and almond consumption in healthy women. Nutr Res 2006;26:509-16. 
42. Moreira Alves RD, Boroni Moreira AP, Macedo VS, et al. Higholeic peanuts: new perspective to attenuate glucose homeostasis disruption and inflammation related obesity. Obesity 2014;22:1981-8.

43. Casas R, Sacanella E, Urpí-Sardà M, et al. The effects of the mediterranean diet on biomarkers of vascular wall inflammation and plaque vulnerability in subjects with high risk for cardiovascular disease. A randomized trial. PLoS One 2014;9:e100084.

44. Casas R, Sacanella E, Urpí-Sardà M, et al. Long-term immunomodulatory effects of a mediterranean diet in adults at high risk of cardiovascular disease in the PREvención con Dleta MEDiterránea (PREDIMED) Randomized Controlled Trial. J Nutr 2016;146:1684-93.

45. Lasa A, Miranda J, Bulló M, et al. Comparative effect of two Mediterranean diets versus a low-fat diet on glycaemic control in individuals with type 2 diabetes. Eur J Clin Nutr 2014;68:767-72.

46. Urpi-Sarda M, Casas R, Chiva-Blanch G, et al. The Mediterranean diet pattern and its main components are associated with lower plasma concentrations of tumor necrosis factor receptor 60 in patients at high risk for cardiovascular disease. $J$ Nutr 2012;142:1019-25.

47. Solà R, Valls RM, Godàs G, et al. Cocoa, hazelnuts, sterols and soluble fiber cream reduces lipids and inflammation biomarkers in hypertensive patients: a randomized controlled trial. PLoS One 2012;7:e31103.

48. Sweazea KL, Johnston CS, Ricklefs KD, et al. Almond supplementation in the absence of dietary advice significantly reduces C-reactive protein in subjects with type 2 diabetes. J Funct Foods 2014;10:252-9.

49. Hu Y, McIntosh GH, Le Leu RK, et al. Supplementation with Brazil nuts and green tea extract regulates targeted biomarkers related to colorectal cancer risk in humans. Br J Nutr 2016;116:1901-11.

50. Rock CL, Flatt SW, Pakiz B, et al. Effects of diet composition on weight loss, metabolic factors and biomarkers in a 1-year weight loss intervention in obese women examined by baseline insulin resistance status. Metabolism 2016;65:1605-13.

51. Barbour JA, Howe PR, Buckley JD, et al. Effect of 12 weeks high oleic peanut consumption on cardio-metabolic risk factors and body composition. Nutrients 2015;7:7381-98.

52. Burns-Whitmore B, Haddad E, Sabaté J, et al. Effects of supplementing n-3 fatty acid enriched eggs and walnuts on cardiovascular disease risk markers in healthy free-living lacto-ovovegetarians: a randomized, crossover, free-living intervention study. Nutr J 2014;13:29.

53. Canales A, Sánchez-Muniz FJ, Bastida S, et al. Effect of walnutenriched meat on the relationship between VCAM, ICAM, and LTB4 levels and PON-1 activity in ApoA4 360 and PON-1 allele carriers at increased cardiovascular risk. Eur J Clin Nutr 2011;65:703-10.

54. Chen CY, Holbrook M, Duess MA, et al. Effect of almond consumption on vascular function in patients with coronary artery disease: a randomized, controlled, cross-over trial. Nutr $J$ 2015;14:61.

55. Chiang YL, Haddad E, Rajaram S, et al. The effect of dietary walnuts compared to fatty fish on eicosanoids, cytokines, soluble endothelial adhesion molecules and lymphocyte subsets: a randomized, controlled crossover trial. Prostaglandins Leukot Essent Fatty Acids 2012;87:111-7.

56. Jenkins DJ, Kendall CW, Marchie A, et al. Dose response of almonds on coronary heart disease risk factors: blood lipids, oxidized lowdensity lipoproteins, lipoprotein(a), homocysteine, and pulmonary nitric oxide: a randomized, controlled, crossover trial. Circulation 2002;106:1327-32.

57. Parham M, Heidari S, Khorramirad A, et al. Effects of pistachio nut supplementation on blood glucose in patients with type 2 diabetes: a randomized crossover trial. Rev Diabet Stud 2014;11:190-6.
58. Rajaram S, Connell KM, Sabaté J. Effect of almond-enriched highmonounsaturated fat diet on selected markers of inflammation: a randomised, controlled, crossover study. Br J Nutr 2010;103:907.

59. West SG, Gebauer SK, Kay CD, et al. Diets containing pistachios reduce systolic blood pressure and peripheral vascular responses to stress in adults with dyslipidemia. Hypertension 2012;60:58-63.

60. Wu L, Piotrowski K, Rau T, et al. Walnut-enriched diet reduces fasting non-HDL-cholesterol and apolipoprotein B in healthy Caucasian subjects: a randomized controlled cross-over clinical trial. Metabolism 2014;63:382-91.

61. Njike VY, Ayettey R, Petraro P, et al. Walnut ingestion in adults at risk for diabetes: effects on body composition, diet quality, and cardiac risk measures. BMJ Open Diabetes Res Care 2015;3:e000115.

62. EFSA Panel on Dietetic Products N, Allergies. Scientific opinion on the substantiation of health claims related to walnuts and maintenance of normal blood LDL-cholesterol concentrations (ID 1156, 1158) and improvement of endothelium-dependent vasodilation (ID 1155, 1157) pursuant to Article 13(1) of Regulation (EC) No 1924/2006. EFSA Journal 2011;9:2074.

63. Thijssen DH, Black MA, Pyke KE, et al. Assessment of flow-mediated dilation in humans: a methodological and physiological guideline. Am J Physiol Heart Circ Physiol 2011;300:H2-H12.

64. Liao JK. Linking endothelial dysfunction with endothelial cell activation. J Clin Invest 2013;123:540-1.

65. Brufau G, Boatella J, Rafecas M. Nuts: source of energy and macronutrients. Br J Nutr 2006;96:S24-8.

66. Lorin J, Zeller M, Guilland JC, et al. Arginine and nitric oxide synthase: regulatory mechanisms and cardiovascular aspects. Mol Nutr Food Res 2014;58:101-16.

67. Cortés B, Núñez I, Cofán M, et al. Acute effects of high-fat meals enriched with walnuts or olive oil on postprandial endothelial function. J Am Coll Cardiol 2006:48:1666-71.

68. Barbaresko J, Koch M, Schulze MB, et al. Dietary pattern analysis and biomarkers of low-grade inflammation: a systematic literature review. Nutr Rev 2013;71:511-27.

69. Ridker PM, Buring JE, Cook NR, et al. C-reactive protein, the metabolic syndrome, and risk of incident cardiovascular events: an 8-year follow-up of 14719 initially healthy American women. Circulation 2003;107:391-7.

70. Del Gobbo LC, Falk MC, Feldman R, et al. Effects of tree nuts on blood lipids, apolipoproteins, and blood pressure: systematic review, meta-analysis, and dose-response of 61 controlled intervention trials. Am J Clin Nutr 2015;102:1347-56.

71. Tapsell LC, Dunning A, Warensjo E, et al. Effects of vegetable consumption on weight loss: a review of the evidence with implications for design of randomized controlled trials. Crit Rev Food Sci Nutr 2014;54:1529-38.

72. Ravussin E, Redman LM, Rochon J, et al. A 2-year randomized controlled trial of human caloric restriction: feasibility and effects on predictors of health span and longevity. J Gerontol A Biol Sci Med Sci 2015;70:1097-104.

73. Dawson JA, Kaiser KA, Affuso O, et al. Rigorous control conditions diminish treatment effects in weight loss-randomized controlled trials. Int J Obes 2016;40:895-8.

74. Barbour JA, Howe PR, Buckley JD, et al. Nut consumption for vascular health and cognitive function. Nutr Res Rev 2014;27:131-58.

75. Neale EP, Batterham MJ, Tapsell LC. Consumption of a healthy dietary pattern results in significant reductions in C-reactive protein levels in adults: a meta-analysis. Nutr Res 2016;36:391-401.

76. Mills EJ, Chan AW, Wu P, et al. Design, analysis, and presentation of crossover trials. Trials 2009;10:27.

77. Estruch R, Martínez-González MA, Corella D, et al. Effects of a Mediterranean-style diet on cardiovascular risk factors: a randomized trial. Ann Intern Med 2006;145:1-11. 